\section{LA SALUTE MENTALE DI BAMBINI E ADOLESCENTI: TEMPO DI AGIRE}

Nel corso dell'ultimo anno della pandemia da Covid-19, una crescente attenzione si è concentrata sulla salute mentale e sul benessere di bambini e adolescenti. Sulle pagine di Medico e Bambino, già nell'aprile 2020, era stato anticipato quello che si è a breve verificato, con una serie di proposte concrete a partire dalla formalizzazione di "un Gruppo multidisciplinare di coordinamento articolato a livello nazionale/o regionale, che possa orientare gli interventi di salute mentale, predisponendo progetti e programmi coerentemente ai bisogni della popolazione e all'andamento della diffusione dell'epidemia sul territorio nazionale"1.

Nel corso di questi lunghi mesi le evidenze dei danni della pandemia sulla salute mentale dei bambini e in particolare degli adolescenti si sono accumulate. Su JAMA Pediatrics è stata pubblicata una metanalisi di Racine e coll. ${ }^{2}$ che richiama l'esigenza di affrontare gli effetti globali sulla salute in questa fascia di popolazione: 29 studi, per un totale di 80.879 casi, documentano che la prevalenza di sintomi rilevanti di depressione e ansia sono significativamente più elevati rispetto a quelli riportate prima dell'inizio della pandemia, a conferma di altri studi sull'argomento ${ }^{3}$. Prima del Covid-19, le stime globali di prevalenza erano dell' $8,5 \%$ per la depressione e dell' 1 1,6\% per l'ansia ${ }^{2,4}$. La metanalisi suggerisce un aumento di oltre 2 volte dei tassi rispetto a quelli riportati prima della pandemia: $23,8 \%$ per la depressione clinicamente significativa e $19 \%$ per l'ansia! ${ }^{2}$. Per completezza si riportano i risultati di una precedente metanalisi che aveva stimato in ventisette Paesi i tassi di prevalenza per i disturbi psichiatrici tra bambini e adolescenti in epoca pre-pandemica, pari al $2,6 \%$ per il disturbo depressivo e al 6,5\% per il disturbo $\mathrm{d}^{\prime}$ ansia ${ }^{4}$.

Gli Autori della metanalisi riportano che la prevalenza di depressione e ansia è aumentata con il progredire della pandemia e che la frequenza dei sintomi è maggiore negli adolescenti e in particolare nelle ragazze ${ }^{2}$. I risultati non sorprendono nel contesto degli straordinari cambiamenti affrontati dai giovani, tra cui l'isolamento sociale, la perdita di interazioni tra pari e le chiusure scolastiche e più in generale della comunità sociale. Questo studio ribadisce in modo inequivocabile che bambini e adolescenti sperimentano un aumento del disagio psicologico e sottolinea l'importanza di una ricerca longitudinale per comprendere se e quanto i sintomi sono mantenuti, esacerbati o risolti nel corso del tempo ${ }^{4}$. Una sorveglianza prospettica condotta in Italia avrebbe dimostrato un miglioramento-risoluzione dei problemi mentali con le riaperture scolastiche ${ }^{5}$.

I limiti di questo tipo di studi, riportati dagli stessi Autori, riguardano il fatto che i lavori si sono poco focalizzati su minoranze etniche e sulle condizioni estreme di disagio. Inoltre, nella quasi totalità dei casi, sono stati utilizzati per la diagnosi strumenti di valutazione basati su questionari auto-somministrati (compilati da parte dei genitori e più raramente degli stessi adolescenti) $)^{2,4}$.

Questi risultati fanno ulteriormente riflettere. In molte Nazioni, tra cui l'Italia (sono in corso valutazioni e verifiche), se si considera la punta dell'iceberg, i bambini e soprattutto gli adolescenti che cercano assistenza nei Servizi di salute mentale, soprattutto quelli che presentano sintomi gravi e richiedono cure a livello ospedaliero sono, in questa fase della pandemia, notevolmente aumentati, in particolare quelli appartenenti a gruppi minoritari ${ }^{6}$.

Per quanto riguarda la prevenzione e la cura, quello che è noto in tutto il mondo è che i Servizi di salute necessari (a partire da quelli che potrebbero essere orientati all'ascolto) sono insufficienti $i^{7,8}$. Sapendo bene che sono disponibili strumenti efficaci per affrontare la depressione e I'ansia (volendo rimanere alle due maggiori condizioni indicate nella metanalisil ${ }^{9}$. Comprendere a livello di popolazione il grado di disagio è oltremodo importante per indirizzare gli interventi appropriati: i giovani con sintomi "sotto-soglia" hanno meno probabilità di richiedere interventi rispetto ai giovani con compromissione funzionale, e intercettarli precocemente può evitare un peggioramento della sintomatologia.

Nonostante queste evidenze, il problema non emerge dai report ufficiali: non c'è voce di questo nei vari Bollettini informativi istituzionali giornalieri e sufficiente attenzione da parte delle direzioni ospedaliere o territoriali. Non esiste una soglia critica di "posti letto occupati" per le conseguenze su bambini e adolescenti degli effetti secondari della pandemia. Basti pensare ai tanti casi ricoverati con disturbi della condotta alimentare.

La pandemia di Covid-19 ci pone di fronte alla necessità di azioni urgenti e incisive, per rispondere in modo efficace ed efficiente a un'emergenza iniziata ben $p \mathrm{rma}^{7,8}$. Occorre sostenere l'implementazione di pratiche supportate da prove e facilitare l'accesso alle cure eliminando le disparità. È necessario innanzitutto rendere visibile il disagio riguardante la salute "psicologica" (termine riduttivo) e la necessità di integrazione sociale dei nostri bambini e adolescenti. Non è questa la sede per fornire modelli validi per una modifica radicale dei paradigmi e dei modi di organizzare I'assistenza. Ne sono stati proposti sia a livello internazionale che nazionale ${ }^{10.13}$. Quello che sappiamo è che sono state stanziate risorse importanti, il cui utilizzo dovrebbe essere guidato da alcuni principi generali così riassumibili:

- rafforzare i Servizi di Neuropsichiatria dell'infanzia e adolescenza su tutto il territorio nazionale, rendendoli adeguati per numero e qualità delle diverse figure professionali necessarie ${ }^{12}$;

- potenziare di molto l'azione preventiva e di diagnosi precoce, a partire dall'ambulatorio del pediatra di famiglia e del medico di Medicina generale ${ }^{14}$, con un lavoro proattivo, singolo e/o di gruppo, di prossimità e/o a distanza, orientato all'ascolto, a consigli e a un complessivo supporto anche della famiglia; quando necessario, all'adozione di strumenti validati in grado di sapere riconoscere le situazioni potenzialmente critiche che possono richiedere, in modo motivato, valutazioni di secondo livello';

- sviluppare l'integrazione e le connessioni nei singoli ambiti distrettuali tra i Servizi sanitari, le scuole, i Servizi di sostegno, i Servizi sociali, attraverso il lavoro di psicologi, educatori e infermieri di comunità ${ }^{13}$. 
L'utilizzo delle risorse destinate alla salute mentale anche dell'infanzia e dell'adolescenza dovrebbe essere guidato da alcuni principi definiti di concerto tra varie figure professionali e organismi istituzionali favorendo, in ogni realtà locale, un collante di ruoli e responsabilità che deve rispondere a una visione fatta di concretezza e progettazione. Il rischio è di reclutare personale (psicologi, educatori, infermieri) per contratti a breve termine, con l'unico obiettivo di evadere le liste di attesa, senza una prospettiva di rete che non insegue i disagi ma che li prevede e previene, con un'assistenza partecipata e modulata per livelli di intensità delle cure.

Fondamentale, in tutto questo, sarà il ruolo della scuola che riapre (con le solite discussioni e i soliti ritardi), e che dovrebbe fornire a bambini e ragazzi una prospettiva di presenza e di qualificata funzione educazionale e di dialogo $^{15}$, uscendo dalle discussioni, a volte stucchevoli, che parlano esclusivamente di rischi, sicurezza e di green pass.

Noi stessi, come pediatri, dovremmo mettere in campo tutte le energie per il lavoro di ascolto e supporto alle famiglie e agli stessi bambini e adolescenti/preadolescenti che sono a noi affidati. A tutti noi, come comunità di operatori sanitari e come singoli cittadini, compete di agire come sentinelle delle singole situazioni, in particolare di quelle con maggiore difficoltà e disagio. II nostro lavoro non può più avere una dimensione solo sanitaria, ma socio-sanitaria ed educativa ${ }^{16,17}$. In ballo c'è il presente e il futuro dei nostri bambini e ragazzi, il cui benessere sociale e psicologico non può che essere al centro di politiche educative, sociali, e certo anche sanitarie, qualificate.

\section{Bibliografia}

1. Abbracciavento G, Cognigni M, Riccio G, Carrozzi M. Covid19 e salute mentale in età evolutiva: I'urgenza di darsi da fare. Medico e Bambino 2020;39(4):237-40.

2. Racine N, McArthur BA, Cooke JE, Eirich R, Zhu J, Madigan S. Global prevalence of depressive and anxiety symptoms in children and adolescents during COVID-19: a meta-analysis. JAMA Pediatr Published online August 9, 2021. doi: 10.1001/jamapediatrics.2021.2482.
3. Minozzi S, Saulle R, Amato L, Davoli M. Impatto del distanziamento sociale per Covid-19 sul benessere psicologico dei giovani: una revisione sistematica della letteratura. Recenti Progr Med $2021 ; 112: 360-70$. doi: 10.1701/3608.35873.

4. Benton TD, Boyd RC, Nioroge WFM. Addressing the global crisis of child and adolescent mental health. JAMA Pediatr Published online August 9, 2021. doi:10.1001/jamapediatrics.2021.2479. 5. Meda N, Pardini S, Slongo I, et al. Students' mental health problems before, during, and atter COVID-19 lockdown in Italy. J Psychiatr $\operatorname{Res} 2021 ; 134$ :69-77. doi: 10.1016/i.jpsychires.2020. 12.045.

6 . Krass P, Dalton E, Doupnik SK, Esposito J. US pediatric emergency department visits for mental health conditions during the COVID-19 pandemic. JAMA Netw Open 2021;4(4):e218533e218533. doi: 10.1001/jamanetworkopen.2021.8533.

7. Marchetti F. I Servizi di salute mentale per i bambini in Inghilterra (e in Italia) "non sono neanche lontanamente sufficienti". Medico e Bambino 2021 ;40(3): 157-8. doi: 10.53126/MEB40157. 8. Gruppo di lavoro per la convenzione dei diritti dell'Infanzia e Adolescenza. Salute Mentale. $11^{\circ}$ Rapporto di Aggiornamento 2020.

9. Kapur N, Ibrahim S, While D, et al. Mental health service changes, organisational factors, and patient suicide in England in 1997-2012: a before-and-after study. Lancet Psychiatry 2016;3 (6):526-534. doi:10.1016/S2215-0366(16)00063-8.

10. Gruppo di lavoro ISS Salute mentale ed emergenza COVID19. Indicazioni ad interim, per un appropriato sostegno della salute mentale nei minori di eta durante la pandemia COVID-19. Versione del 31 maggio 2020. Roma: Istituto Superiore di Sanita; 2020. (Rapporto ISS COVID-19 n. 43/2020).

11. AAP. Mental Health During Covid-19: Signs Your Child May Need More Support. Last updated 30/7/2021.

12. Ministero della Salute. Linee di indirizzo sui disturbi neuropsichiatrici e neuropsichi-ci dell'infanzia e della adolescenza. Ultimo aggiornamento 3 giugno 2021 .

13. Centro per la Salute del Bambino, Associazione Culturale Pediatri. Senza confini: come ridisegnare le cure per l'infanzia e l'adolescenza, integrando i servizi, promuovendo l'equità, diffondendo le eccellenze- 2a versione, pubblicato on-line, 12.05.2021. 14. Tamburlini $G$, Volta A. Il bambino tutto intero: per un approccio integrato al bambino e al suo ambiente complesso. Medico e Bambino 2021;40(4):237-44. doi: 10.53126/MEB40237.

15. Bonati M. Scuole in sicurezza. Ric\&Pra $2021 ; 17(4): 147-9$. doi: $10.1707 / 3657.36396$.

16. Liverani A, Ercolanese TI, Valletta E. Adolescenti nella nicchia. Medico e Bambino 2021;40(6):401-4. doi: 10.53126/ MEB4040 1

17. Marchetti F. La voce dei "minori" durante e dopo la pandemia. Recenti Prog Med 2021;112(5):331-4. doi: 10.1701/3608. 35868 .

Federico Marchetti 